\title{
Effect of iron-doping on spin-state transition and ferromagnetism in $\mathrm{Pr}_{0.5} \mathrm{Ca}_{0.5} \mathrm{CoO}_{3-\delta}$ cobalt oxides
}

\author{
X. G. Luo, X. Li, G. Y. Wang, G. Wu and X. H. Chen* \\ Hefei National Laboratory for Physical Science at Microscale and Department of Physics, \\ University of Science and Technology of China, \\ Hefei, Anhui 230026, People's Republic of China
}

\begin{abstract}
Resistivity and dc magnetization measurements were performed for the polycrystalline $\operatorname{Pr}_{0.5} \mathrm{Ca}_{0.5} \mathrm{Co}_{1-x} \mathrm{Fe}_{x} \mathrm{O}_{3-\delta}(x=0,0.05,0.10$ and 0.15$)$ samples. The as-fabricated samples exhibit ferromagnetic (FM) transition and the transition temperature increases with increasing the iron doping level. Annealing under high oxygen pressure induces a spin-state transition of Co ions in the iron-free sample and such transition is reinforced with increasing the annealing oxygen pressure, while the annealing under high oxygen pressure suppresses the ferromagnetic ordering. Contrary to the case of the iron-free sample, no spin-state transition is induced by the annealing under high oxygen pressure for the iron-doped samples, and the ferromagnetic transition temperature is nearly independent of the annealing procedures. The enhancement of the spin-state transition in the ironfree sample after annealing under high oxygen pressure should be attributed to the reduction of the cell volume. The suppression of the spin-state transition by the Fe doping is related to the enlargement of the cell volume and the stronger Fe-O bonds than Co-O bonds. The enhancement of the ferromagnetism by the iron-doping might arise from the ferromagnetic exchange interaction between $\mathrm{Fe}^{3+}$ and $\mathrm{Co}^{4+}$ through oxygen $\left(\mathrm{Fe}^{3+}-\mathrm{O}-\mathrm{Co}^{4+}\right)$.

PACS numbers: 75.30.-m,71.30.+h,71.70.-d,75.47.-m
\end{abstract}

\footnotetext{
${ }^{*}$ Corresponding author; Electronic address: chenxh@ustc.edu.cn
} 


\section{INTRODUCTION}

An intriguing feature of the perovskite-related cobalt oxides is the existence of spin-state transition (SST), which can be induced by temperature, pressure, or carrier concentration. There are various spin states for trivalent (low-spin LS: $t_{2 \mathrm{~g}}^{6} e_{g}^{0}$; intermediate-spin IS: $t_{2 g}^{5} e_{g}^{1}$; high-spin HS: $t_{2 g}^{4} e_{g}^{2}$ ) and tetravalent cobalt ions (LS: $t_{2 g}^{5} e_{g}^{0}$; IS: $t_{2 g}^{4} e_{g}^{1}$; HS: $t_{2 g}^{3} e_{g}^{2}$ ). In the simplest perovskite-type $\mathrm{LnCoO}_{3}(\mathrm{Ln}=\mathrm{Y}$, and rare earth elements), a transition from lowspin to high- or intermediate-spin was observed with increasing the temperature. [1, 2, 3, 4, 4, 5] In another interesting oxygen-deficient cobalt oxides $\mathrm{LnBaCo}_{2} \mathrm{O}_{5+\delta}(\mathrm{Ln}=\mathrm{Pr}, \mathrm{Nd}, \mathrm{Sm}, \mathrm{Eu}$, Gd and $\mathrm{Tb}$ ), an abrupt SST was reported as $\delta=0.5$. [6, 7, [8, 9, 10] The conversion of different spin-state arises from the competition between comparable the crystal-field with energy $\Delta_{\mathrm{CF}}$ $\left(t_{2 \mathrm{~g}}-e_{\mathrm{g}}\right.$ splitting) and the intraatomic (Hund) exchange with energy $J_{\text {ex }}$ in magnitude, leading to redistribution of electrons between $t_{2 \mathrm{~g}}$ and $e_{\mathrm{g}}$ levels. $\Delta_{\mathrm{CF}}$ is found to be very sensitive to the variation in the Co-O bond length $\left(d_{\mathrm{Co}-\mathrm{O}}\right)$, so that the subtle balance between $\Delta_{\mathrm{CF}}$ and $J_{\text {ex }}$ may be easily disrupted by different kind of effects such as hole-doping and chemical or

external pressure. [11, 12, 13, 14] Very recently, Lengsdorf er al. and Fita et al. reported that the pressure suppressed FM interactions in $\mathrm{La}_{0.82} \mathrm{Sr}_{0.18} \mathrm{CoO}_{3}$ and $\mathrm{La}_{1-x} \mathrm{Ca}_{x} \mathrm{CoO}_{3}$ due to the reduction of the population of the $e_{g}$ electrons induced by the increase in the energy of crystal field splitting. [13, 14]

The transition of the spin state of cobalt ions is always connected with the change of conductivity. The metal-insulator transition (MIT) is usually accompanied with a SST. A metal to insulator transition in resistivity occurs in $\mathrm{LaCoO}_{3}$ as the spin-state changes gradually from high-spin to low-spin state between $350 \mathrm{~K}$ to $110 \mathrm{~K}$. 15] In $\mathrm{LnBaCo}_{2} \mathrm{O}_{5+\delta}$ $(\mathrm{Ln}=\mathrm{Pr}, \mathrm{Nd}, \mathrm{Sm}, \mathrm{Eu}, \mathrm{Gd}$ and $\mathrm{Tb})$, an abrupt MIT takes place simultaneously as the SST occurs. 6, 7, 8, 9, 10] The occurrence of MIT accompanying with the SST suggests the essential role of the $e_{g}$ electrons in the metallic electronic conduction.

In the present paper, we paid our attention on another cobalt oxide $\operatorname{Pr}_{0.5} \mathrm{Ca}_{0.5} \mathrm{CoO}_{3-\delta}$, which was reported firstly by Tsubouchi et al. 16, 17] to exhibit the simultaneous MIT and SST at low temperature. They revealed that the MIT and SST in $\operatorname{Pr}_{0.5} \mathrm{Ca}_{0.5} \mathrm{CoO}_{3-\delta}$ arise from the large decrease of Co-O-Co angle around the transition temperature with decreasing temperature. The large decrease of Co-O-Co angle results in an extraction of lattice volume and a reduction in the covalence of the Co-O bonds, which enlarges the splitting of the 
crystal field and stabilizes the localized low-spin state. As one knows, the ferromagnetic exchanges occur mainly through the $e_{\mathrm{g}}$ hopping in perovskite-type cobalt oxides. The reduction in the population of the $e_{\mathrm{g}}$ electrons has an effect of suppressing ferromagnetism, which has been demonstrated by previous researches of using external pressure [13, 14] or chemical pressure. [18, 19] Therefore, the degradation of spin-state of cobalt ions would suppress the $e_{g}$ hopping and consequently the ferromagnetic interaction. Actually, Tsubouchi et al. [16, 17] presented that the long ferromagnetic order does not appear down to $10 \mathrm{~K}$ in $\mathrm{Pr}_{0.5} \mathrm{Ca}_{0.5} \mathrm{CoO}_{3}$. It seems to suggest that the spin-state transition is competitive with the ferromagnetism. Thus, if ferromagnetism is enhanced through some ion substitution, spin-state transition is expected to be suppressed. Maignan et al. proposed that the superexchange between the high-spin $\mathrm{Fe}^{3+}$ and the low-spin $\mathrm{Co}^{4+}$ ions through the oxygen atom

could be ferromagnetic. [20] In this work, we examined effect of Fe doping and annealing procedure under the high oxygen pressure (HP) on the ferromagnetism, spin-state transition, and charge transport in the polycrystalline $\mathrm{Pr}_{0.5} \mathrm{Ca}_{0.5} \mathrm{Co}_{1-x} \mathrm{Fe}_{x} \mathrm{O}_{3-\delta}$ samples. The results indicate that the ferromagnetism is enhanced, while spin-state transition is suppressed by the Fe doping. On the other hand, the annealing procedures under the high oxygen pressure induce a spin-state transition in the iron-free sample, but do not change the ferromagnetic transition temperature in the iron-doped samples.

\section{EXPERIMENTAL PROCEDURES}

The polycrystalline ceramic $\mathrm{Pr}_{0.5} \mathrm{Ca}_{0.5} \mathrm{Co}_{1-x} \mathrm{Fe}_{x} \mathrm{O}_{3-\delta}$ samples with $x=0,0.05,0.10$ and 0.15 were synthesized using conventional solid state reaction meathod. They were prepared from a stoichiometric mixture of the fine powder of oxides: $\mathrm{Pr}_{6} \mathrm{O}_{11}, \mathrm{CaCO}_{3}, \mathrm{Co}_{2} \mathrm{O}_{3}$ and $\mathrm{Fe}_{2} \mathrm{O}_{3}$. The stoichiometric mixture was ground carefully until the mixture became homogeneous. The mixtures then were calcined at $1200^{\circ} \mathrm{C}$ in the flowing oxygen for $24 \mathrm{~h}$. The calcined powder was reground and pressed into pellets. The pellets were sintered at $1200^{\circ} \mathrm{C}$ in the flowing oxygen for $24 \mathrm{~h}$ and finally cooled to room temperature. Some portions of pellets for each composition were annealed at $600^{\circ} \mathrm{C}$ for $48 \mathrm{~h}$ under the oxygen pressure of $115 \mathrm{~atm}, 175 \mathrm{~atm}$ and $280 \mathrm{~atm}$, respectively. The X-ray powder diffraction (XRD) was recorded at room temperature using X Pert PRO X-Ray diffractometer (Philips) with $\mathrm{CuK} \alpha$ radiation $(\lambda=1.5418 \AA)$. The resistivity measurements were performed using the standard 
ac four-probe method. The magnetic field was supplied by a superconducting magnet system (Oxford Instruments). Magnetization measurement was carried out with a superconducting quantum interference device (SQUID) magnetometer (MPMS-7XL, Quantum Design). We also determined the oxygen content of the samples using $\mathrm{K}_{2} \mathrm{Cr}_{2} \mathrm{O}_{7}$ titration method. An appropriate amount of sample (about $30 \mathrm{mg}$ ) is dissolved in the mixture of vitriol and phosphate acid, then the high valent Co ions are deoxidized to divalent ones with $\mathrm{Fe}^{2+}$ ions, and finally the excess $\mathrm{Fe}^{2+}$ ions are titrated with $\mathrm{K}_{2} \mathrm{Cr}_{2} \mathrm{O}_{7}$ solution. The oxygen content for the as-fabricated samples was determined as $2.930 \pm 0.005$. The oxygen content increases after annealing under the oxygen pressure, and changes to $2.959 \pm 0.005$ at $115 \mathrm{~atm}, 2.978 \pm 0.003$ at 175 atm and $2.990 \pm 0.002$ at 280 atm, respectively.

\section{EXPERIMENTAL RESULTS}

\section{A. Structural charactization}

In Fig. 1, the unit cell volumes of the as-fabricated $\mathrm{Pr}_{0.5} \mathrm{Ca}_{0.5} \mathrm{Co}_{1-x} \mathrm{Fe}_{x} \mathrm{O}_{3-\delta}(x=0,0.05$, 0.10 and 0.15 ) samples determined by the XRD patterns at room temperature are plotted against $x$. In the determination of the lattice parameters, the orthorhombic structural symmetry is assumed and the volume is estimated for the cell described by $\sqrt{2} a_{\mathrm{p}} \times 2 a_{\mathrm{p}} \times$ $\sqrt{2} a_{\mathrm{p}}$ (space group Pnma) with $a_{\mathrm{p}}$ being the lattice constant of the cubic perovskite cell. Such a crystallographic structure has been used in $\operatorname{Pr}_{1-x} \mathrm{Ca}_{x} \mathrm{CoO}_{3}$ system previously by other anthors. 16, 17, 22] The volume increases with $x$, because ionic radius of $\mathrm{Fe}^{3+}$ is

slightly larger than that of $\mathrm{Co}^{3+}$ and $\left.\mathrm{Co}^{4+} .23,24\right]$ The unit cell volumes of the iron-free sample is also shown in Fig. 1 with varying the annealing oxygen pressure. The volume decreases with increasing oxygen pressure (oxygen content), similar to the results observed in $\mathrm{La}_{0.5} \mathrm{Sr}_{0.5} \mathrm{CoO}_{3-\delta} \cdot[21]$

\section{B. The magnetic and transport properties for the as-fabricated samples}

Figure 2 shows the temperature dependence of the zero field cooled (ZFC) and field cooled (FC) molar magnetization $M$ at $H=0.1 \mathrm{~T}$ for the as-fabricated $\mathrm{Pr}_{0.5} \mathrm{Ca}_{0.5} \mathrm{Co}_{1-x} \mathrm{Fe}_{x} \mathrm{O}_{3-\delta}(x$ $=0,0.05,0.10$ and 0.15$)$ samples. It clearly shows that all samples undergo a ferromagnetic transition at the $T_{\mathrm{c}}$ (taken at the inflection point of the FC curve) which is enhanced from 
about $77 \mathrm{~K}$ to $86 \mathrm{~K}$ with increasing $x$ from 0 to 0.15 .The iron-doped samples exhibit almost the same FC $M$ below $50 \mathrm{~K}$, their molar magnetization is much larger that that of the Fe-free sample. As shown in Fig.2, the magnetic moment of the Fe-free sample at $4 \mathrm{~K}$, is $2470 \mathrm{emu} / \mathrm{mol}\left(\approx 0.44 \mu_{\mathrm{B}} / \mathrm{Co}\right)$, while it increases to $2950 \mathrm{emu} / \mathrm{mol}\left(\approx 0.53 \mu_{\mathrm{B}} / \mathrm{Co}\right)$ by the Fe doping. From the $M-H$ loops collected at $4 \mathrm{~K}$ for these four samples (shown Fig. 3), obvious spontaneous magnetization $\left(M_{\mathrm{s}}\right)$ and clear hysteresis are observed, consistent with the ferromagnetic transition. A pronounced characteristics in Fig.3 is that the $M_{\mathrm{s}}$ increases with the iron doping level. In contrast to the sudden enhancement of spontaneous magnetization with increasing $x$ from 0 to 0.05 , a slight increase in the $M_{\mathrm{s}}$ is observed with further increasing $x$, in accord with the results observed in Fig.2. Apparently, the Fe doping enhances the ferromagnetism. Another marked feature can be found in Fig. 3 is that the three iron-doped samples have the same coercive force of 2535 Oe while the iron-free sample shows a much larger coercive force of 3475 Oe.

Figure 4 presents the resistivity, $\rho$, as a function of temperature for the as-fabricated $\mathrm{Pr}_{0.5} \mathrm{Ca}_{0.5} \mathrm{Co}_{1-x} \mathrm{Fe}_{x} \mathrm{O}_{3-\delta}(x=0,0.05,0.10$ and 0.15$)$ samples. The resistivity grows monotonically with increasing the iron doping level. All samples shows insulating behavior in the high temperatures. With decreasing temperature, the resistivity for all samples exhibits the change of slope at a temperature of $T_{\mathrm{p}}$, and a metal-insulator transition occurs at $T_{\mathrm{p}}$ for the samples with $x=0$ and 0.05 . The values of $T_{\mathrm{p}}$ are $74.3,80.2,82.0$, and 85.7 for $x=0,0.0 .5$, 0.10 and 0.15 , respectively. It is found that the $T_{\mathrm{p}}$ is nearly the same as the $T_{\mathrm{c}}$. In addition, the variation of $T_{\mathrm{p}}$ with Fe doping coincides with that of $T_{\mathrm{c}}$. These suggests that the inflection of the resistivity is induced by the reduction of the spin scattering due to the ferromagnetic transition. In spite of the increase of $T_{\mathrm{p}}$ and $T_{\mathrm{c}}$, the conductivity decreases with increasing iron doping level. An increase by only four times in $\rho$ was observed at $4 \mathrm{~K}$ with increasing $x$ from 0 to 0.15 , however, an increase in $\rho$ by over seven orders at $77 \mathrm{~K}$ occurred with iron doping from 0 to 0.15 in the manganites in $\mathrm{Nd}_{0.67} \operatorname{Sr}_{0.33} \mathrm{Mn}_{1-x} \mathrm{Fe}_{x} \mathrm{O}_{3}$. [25] When a magnetic field is applied, the resistivity is suppressed dramatically at low temperatures as shown in Fig. 5. It is the typical behavior in an itinerant ferromagnet and the resistivity is reduced by magnetic field due to the suppression of spin-scattering. The magnetoresistance $\Delta \rho / \rho=[\rho(H)-\rho(0)] / \rho$ at $H=6 \mathrm{~T}$ is also shown in Fig. 5. Negative $\Delta \rho / \rho$ and a peak of $\Delta \rho / \rho$ around $T_{\mathrm{p}}$ are observed for all the samples. As $x$ increases, the magnitude of the peak is reduced gradually. On the contrary, the magnitude of $\Delta \rho / \rho$ at lower temperatures 
is enhanced by increasing the iron content. The decrease in magnitude of $\Delta \rho / \rho$ is induced from the enhancement of ferromagnetism, because the reduction of spin-scattering by the external magnetic field is smaller in a stronger ferromagnet. Deduced from the increase of resistivity with the Fe doping in the Fig. 4, it can be concluded that the Fe doping in this cobalt oxide should induce disorder as well as the enhancement of ferromagnetism, probably due to randomly distribution of iron ions. This could be considered as the reason of the increase in magnitude of $\Delta \rho / \rho$ at $4 \mathrm{~K}$.

\section{The transport and magnetic properties for the post-annealed samples}

Figure 6 displays the temperature dependence of the resistivity for the $\mathrm{Pr}_{0.5} \mathrm{Ca}_{0.5} \mathrm{Co}_{1-x} \mathrm{Fe}_{x} \mathrm{O}_{3-\delta}(x=0,0.05,0.10$ and 0.15$)$ samples after annealing at $600^{\circ} \mathrm{C}$ under the oxygen pressure of $115 \mathrm{~atm}$. It can be found that the conductivity for the samples with $x=0.10$ and 0.15 is enhanced, while the conductivity for the samples with $x=0$ and 0.05 is reduced, relative to the as-fabricated samples. Especially, the resistivity for the sample with $x=0$ exhibits a rapid increase below about $75 \mathrm{~K}$, an increase by almost 10 times in resistivity at $4 \mathrm{~K}$ relative to that at $75 \mathrm{~K}$ is observed. Similar behavior in the iron-free sample have been observed in previous reports [16, 17] and is attributed to the occurrence of a transition from high-temperature IS state to low-temperature LS state. However, the change in resistivity from the transition point to $4 \mathrm{~K}$ (about ten times) is much less than that observed in the reports (more than three orders in magnitude). 16, 17] It is noted that such a transition is induced after annealing under the high oxygen pressure. Consequently, higher oxygen pressure annealing procedures were carried out.

Figure 7 shows the resistivity as a function of temperature for the $\operatorname{Pr}_{0.5} \mathrm{Ca}_{0.5} \mathrm{Co}_{1-x} \mathrm{Fe}_{x} \mathrm{O}_{3-\delta}$ $(x=0,0.05,0.10$ and 0.15$)$ samples after annealing at $600^{\circ} \mathrm{C}$ under the oxygen pressure of $175 \mathrm{~atm}$. Although the resistivity of the sample with $x=0.15$ shows very slight change, the resistivity for the samples with $x=0.05$ and 0.10 decreases apparently relative to the samples annealing under the oxygen pressure of $115 \mathrm{~atm}$. Nevertheless, the temperaturedependent behavior is almost unchanged. A strange thing can be found that the temperature corresponding to the inflection of the resistivity $\left(T_{\mathrm{p}}\right)$ remains unchanged with the annealing conditions. This suggests that the ferromagnetic transition temperature for the Fe-doped samples is independent with the variation of the annealing conditions. Figure 7 indicates 
that the resistivity for the iron-free sample increases by more than 30 times with decreasing temperature from $70 \mathrm{~K}$ to $4 \mathrm{~K}$, much larger than that in the sample annealed under the oxygen pressure of $115 \mathrm{~atm}$. This confirms that annealing under the high oxygen pressure can enhance the spin-state transition.

Although the spin-state transition has been manifested apparently in the resistivity behavior in the iron-free sample after annealing under the oxygen pressure of $175 \mathrm{~atm}$, Figure 8 shows that there is still no rapid drop in magnetization around the spin-state transition temperature, which has been observed in previous reports. 16, 17] Nevertheless, the magnetization for the iron-free sample after annealing decreases apparently compared to that shown in Fig. 2, indicating that the magnetic moment per cobalt $\left(0.24 \mu_{\mathrm{B}} /\right.$ Co at $\left.4 \mathrm{~K}\right)$ decreases dramatically. This is consistent with the occurrence of the spin-state transition. Actually, the magnitude of $\rho$ above $75 \mathrm{~K}$ for the iron-free sample increases with increasing annealing oxygen pressure (see Fig. 6 and Fig.7) could suggest the depopulation of $e_{g}$ electrons in the iron-free sample even at room temperatures since the conduction is mainly dominated by the transfer of $e_{\mathrm{g}}$ electrons. The iron-doped samples still behave as a good ferromagnet with the same $T_{\mathrm{c}}$ as that in Fig. 2. This is consistent with the results $\left(T_{\mathrm{p}}\right)$ indicated by the resistivity. However, one should note that the magnetization at $4 \mathrm{~K}$ increases for the samples with $x=0.10$ and 0.15 after annealing compared to the as-fabricated samples, on the contrary, the $M(T)$ for the sample with $x=0.05$ decreases. This suggests that the degradation of the spin state in the sample with $x=0.05$ is partly induced by annealing procedure under the high oxygen pressure. Similar to the iron-free sample, the resistivity of the sample with $x=0.05$ becomes larger than that of the sample with $x=0.10$ after annealing under high oxygen pressure (see Fig. 6, Fig. 7), and this is consistent with the partly induced degradation of spin-state in the sample with $x=0.05$ by annealing procedure deduced from Fig. 8. The $M-H$ loops collected at $4 \mathrm{~K}$ for these four samples are shown in Fig. 9. At first glance, it is noted that the three iron doped samples exhibits the same coercive force of 2410 Oe, similar to that observed in the as-fabricated samples but slightly less. The iron-free sample shows a smaller coercive force than those observed in the iron-doped samples. The apparent spontaneous magnetization and the clear hysteresis loop evidence the existence of ferromagnetic order in all of these four samples. The enhancement of $M_{\mathrm{s}}$ with increasing iron doping level is observed, similar to that in Fig. 3. Much lower $M_{\mathrm{s}}$ $\left(0.33 \mu_{\mathrm{B}}\right)$ at $4 \mathrm{~K}$ is obtained in Fig. 9 for the iron-free sample than that in Fig. 4, evidencing 
the degradation of spin-state of cobalt ions and consistent with the results in Fig. 8.

In attempt to obtain the iron-free sample with an abrupt drop in $M(T)$ at the spin-state transition temperature as observed in literatures, [16, 17] further annealing procedure under the oxygen pressure of $280 \mathrm{~atm}$ is performed. Figure 10 shows the FC magnetization at $H$ $=0.1 \mathrm{~T}$ and the resistivity at zero field as the function of temperature. Larger enhancement in magnitude of resistivity from $75 \mathrm{~K}$ to $4 \mathrm{~K}$ is obtained (compared to Fig. 6 and Fig. 7), at the same time, a cusp can be observed in $M(T)$ curve at $66 \mathrm{~K}$, corresponding to midpoint of the sharp increase of resistivity as shown in Fig. 10. Therefore, it can be concluded that annealing under the high oxygen pressure promotes the spin-state transition in the iron-free sample.

\section{DISCUSSIONS}

A most intriguing result in the present work is the enhancement of the SST by the annealing procedure under the high oxygen pressure in the iron-free sample. For the asfabricated iron-free sample, there is no obvious indication for a SST, while after annealing under the oxygen pressure of 115 atm and 175 atm, the resistivity exhibits MIT below 70 $\mathrm{K}$, which is considered to be related to a SST, and at the same time, the magnetization at low temperature is reduced dramatically (see Fig. 11(a)). After annealing under the oxygen pressure of $280 \mathrm{~atm}$, a clear SST similar to that reported by other authors [16, 17] is observed in the $M(T)$ curve. We have demonstrated in Sec. II that the oxygen content is enhanced with increasing the oxygen pressure in the annealing procedure. It is noted that the SST in $\operatorname{Pr}_{0.5} \mathrm{Ca}_{0.5} \mathrm{CoO}_{3-\delta}$ takes place in a system including nearly 50 : 50 trivalent and tetravalent Co ions. At first glance, one may argue that nearly 50 : 50 trivalent and tetravalent Co ions be essential for the occurrence of the SST. However, Fujita et al. 22, 26] reported that the similar SST occurs in $\left(\operatorname{Pr}_{1-y} \mathrm{R}_{y}^{\prime}\right)_{0.7} \mathrm{Ca}_{0.3} \mathrm{CoO}_{3}\left(\mathrm{R}^{\prime}=\mathrm{Sm}, \mathrm{Tb}, \mathrm{Y}\right)$. Therefore, the proportion of nearly 50 : 50 trivalent and tetravalent Co ions is not necessary. As one knows, the spin state of Co ions is mainly determined by the competition in magnitude between $\Delta_{\mathrm{CF}}$ and $J_{\mathrm{ex}}$, which controls the difference of the electronic energies, $\Delta E$, between the spin states. Fig. 1 shows that the cell volume decreases with increasing the oxygen content. The reduction of cell volume can be induced by the decrease of the Co-O-Co angle or the shrink of the Co-O band length. The former reduces the Co-Co transfer energy $t$ and the latter enhances the 
$\Delta_{\mathrm{CF}}$. Both effects favor to stabilize a low-spin state. Therefore, the reduction of the cell volume due to the increase of the oxygen content may be responsible for the enhancement of SST by annealing procedure under the high pressure oxygen.

It is apparent that the Fe doping in $\operatorname{Pr}_{0.5} \mathrm{Ca}_{0.5} \mathrm{CoO}_{3-\delta}$ suppresses the spin-state transition. Even after annealing under the oxygen pressure of $175 \mathrm{~atm}$, clear ferromagnetic transition is observed in the sample with $x=0.05$, although the magnetization is reduced relative to that in as-fabricated one (see Fig. 11 (b)). This suggests that the Fe doping has the effect of destroying the mechanism for the occurrence of SST. $\Delta E$ is dependent on the volume of the $\mathrm{CoO}_{6}$ octahedra and the Co-Co transfer energy $t$. The slight expansion of the unit cell volume with the Fe doping is observed (see Fig. 1). This could result from either the enlargement of the $\mathrm{CoO}_{6}$ octahedra or the decrease of the $\mathrm{CoO}_{6}$ octahedra tilt, i.e. the increase of the Co-O-Co angle. The former possibility would reduce the $\Delta_{\mathrm{CF}}$ and the latter one would enhance $t$. Both of these possible effects diminish the $\Delta E$ and suppress the tendency of spin-state transition. Furthermore, Katsuki et al.[27] presented that Fe-O bonds are stronger than Co-O bonds. The strong covalency of Fe-O bonds broadens the energy widths of the $e_{\mathrm{g}}$ bands through competing with the Co-O bonds and consequently leads to a narrowing of the energy gap $\Delta E$, which could enhance the population of $e_{\mathrm{g}}$ electrons and stabilize an intermediate-spin state.

The electrical transport is dramatically affected by the impact on the spin-state transition from the annealing procedure under high oxygen pressure and the Fe doping. In the asfabricated samples, the resistivity increases with increasing the Fe doping level in spite of the enhancement of the ferromagnetism, which has been attributed to the disorder induced by the Fe doping. While after annealing under the high oxygen pressure of 115 atm, the evolution of resistivity changes dramatically (see Fig. 6). Contrary to the decrease of resistivity in the samples of $x=0.10$ and 0.15 due to the increase of the carrier concentration (oxygen content), the resistivity increases for the samples of $x=0$ and $x=0.05$ and becomes larger than that of the samples with higher Fe doping level (at least at room temperature). As further annealing procedure under $175 \mathrm{~atm}$ is performed, the magnitude of resistivity of the sample with $x=0.05$ decreases and becomes to lie between those of the samples with $x$ $=0.10$ and 0.15 , while that for the iron-free sample keeps to increase still. As one knows, the transfer of the $e_{\mathrm{g}}$ electrons determines the conduction in the perovskite-type cobalt oxides. The enhancement of oxygen content leads to the increase of carrier concentration, and this 
causes the decrease of the resistivity with increasing annealing pressure in the ferromagnetic samples with $x=0.10$ and 0.15 . While in the samples with $x=0$ and 0.05 , besides the increase of hole concentration, it has been pointed out that SST (partly for $x=0.05$ ) is induced by the increase of oxygen content, suggesting the depopulation of $e_{\mathrm{g}}$ electrons. This leads to the increase of resistivity in these two samples. For the two samples, SST is much stronger for the iron-free sample, so that the resistivity keeps increasing with the increase of the annealing oxygen pressure. While the SST in the sample with $x=0.05$ occurs only partly, so that the increase of carrier concentration still can lower the resistivity, which makes the resistivity of this sample firstly increases after annealing under $115 \mathrm{~atm}$ then decreases after further annealing under 175 atm (see Fig. 6 and Fig. 7). Therefore, it can be turned out that the corporate effects of the disorder from the Fe doping, the change of carrier concentration, and spin-state transition lead to the intriguing evolution of the resistivity with the annealing procedures.

Another interesting result induced by the iron doping is the enhancement of the ferromagnetism. As discussed above, an apparent result of the Fe doping is the suppression of the SST. So a possible candidate responsible for the enhancement of the ferromagnetism may be the enhancement of spin state of Co ions, which means the presence of more $e_{\mathrm{g}}$ electrons. However, Fig. 11(b) indicates that although the magnetization for the sample with $x=$ 0.05 decreases with increasing the annealing oxygen pressure, indicative of the existence of a partly induced SST in this sample, the $T_{\mathrm{c}}$ remains unchanged before and after annealing. For the samples with $x=0.10$ and 0.15 the magnetization increases with increasing the annealing oxygen pressure, but the $T_{\mathrm{c}}$ also remains unchanged before and after annealing (See Fig. 2 and Fig. 8). Therefore, it suggests that the rise of spin state due to the Fe doping could not be the reason of the enhancement of the ferromagnetism. According to the studies of Mössbauer spectrum in the iron-doped $\mathrm{TbBaCo}_{2} \mathrm{O}_{5.5}$ and $\mathrm{La}_{1-x} \mathrm{Sr}_{x} \mathrm{CoO}_{3}$, 28, 29, 30] the iron ions have the formation of $\mathrm{Fe}^{3+}$ with high-spin $t_{2 \mathrm{~g}}^{3} e_{\mathrm{g}}^{2}$ electronic configuration. If we assume the same state of iron ions also exists in the present compositions, the ferromagnetic superexchange between $\mathrm{Fe}^{3+}-\mathrm{O}-\mathrm{Co}^{4+}$ in the $\mathrm{LS} \mathrm{Co}^{4+}$ ions could be expected. [20] This would enhance the ferromagnetism of system. 


\section{CONCLUSION}

In summary, a detailed study of the magnetic and transport properties in $\operatorname{Pr}_{0.5} \mathrm{Ca}_{0.5} \mathrm{Co}_{1-x} \mathrm{Fe}_{x} \mathrm{O}_{3-\delta}(\mathrm{x}=0,0.05,0.10$ and 0.15$)$ polycrystalline bulks has been done. Although no apparent structural change is associated with the iron doping, ferromagnetism is systematically enhanced while conductivity is suppressed for the as-fabricated samples. Annealing under high pressure oxygen induces a spin state transition in the iron-free sample, and the SST become more obvious with increasing the oxygen pressure. Fe doping suppresses the SST and enhances the ferromagnetic transition. The SST induced by annealing under

high oxygen pressure in the iron-free sample is attributed to the reduction of cell volume due to the enhancement of the oxygen content. The enlargement of the cell volume induced by the Fe doping and the stronger Fe-O bonds than Co-O bonds are considered to lead to the suppression of SST in the iron-doped samples. The existence of possible ferromagnetic superexchange between $\mathrm{Fe}^{3+}{ }_{-} \mathrm{O}-\mathrm{Co}^{4+}$ is taken into account to understand the enhancement of ferromagnetism by the Fe doping.

\section{ACKNOWLEDGEMENT}

This work is supported by the grant from the Nature Science Foundation of China and by the Ministry of Science and Technology of China, and the Knowledge Innovation Project of Chinese Academy of Sciences. 
[1] S. Yamaguchi, Y. Okimoto, and Y. Tokura, Phys. Rev. B 54, R11022 (1996).

[2] L. Sudheendra, M. M. Seikh, A. R. Raju, and C. Narayana, Chem. Phys. Lett. 340, 275 (2001).

[3] J. Hejtmánek, Z. Jirák, K. Knížek, M. Maryško, M. Veverka, and H. Fujishiro, J. Magn. Magn. Mat. 272-276, E283 (2004).

[4] J. Q. Yan, J. S. Zhou, and J. B. Goodenough, Phys. Rev. B 69, 134409 (2004).

[5] K. Knížek, Z. Jirák, J. Hejtmánek, M. Veverka, M. Marysko, G. Maris and T. T. M. Palstra, cond-mat/0503104 (2005).

[6] N. V. Kasper, I. O. Troyanchuk, D. D Khalyavin, N. Hamad, L. Haupt, P. Frobel, K. Barner, E. Gmelin, Q. Huang, and J. W. Lynn, Physica Status Solidi B 215, 697 (1999).

[7] Y. Moritomo, T. Akimoto, M. Takeo, A. Machida, E. Nishibori, M. Takata, M. Sakata, K. Ohoyama, and A. Nakamura, Phys. Rev. B 61, R13325-R13328 (2000).

[8] D. Akahoshi, and Y. Ueda, J. Solid State Chem. 156, 355 (2001).

[9] C. Frontera, J. L. Garcia-Munoz, A. Llobet, and M. A. G. Aranda, Phys. Rev. B 65, 180405 (2002).

[10] C. Frontera, J.L. Garcia-Munoz, A . Llobet, M. A. G. Aranda, J. Rodriguez-Carvajal, M. Respaud, J.M. Broto, B. Raquet, H. Rakoto, M. Goiran, J. Magn. Magn. Mater. 242-245, $751(2002)$.

[11] K. Asai, O. Yokokura, M. Suzuki, T. Naka, T. Matsumoto, H. Takahashi, N. Mori, and K. Kohn, J. Phys. Soc. Jpn. 66, 967 (1997); K. Asai, A. Yoneda, O. Yokokura, J. M. Tranquada, G. Shirane, and K. Kohn, ibid. 67, 290 (1998).

[12] T. Vogt, J. A. Hriljac, N. C. Hyatt, and P. Woodward, Phys. Rev. B 67, 140401 (2003).

[13] R. Lengsdorf, M. Ait-Tahar, S. S. Saxena, M. Ellerby, D. I. Khomskii, H. Micklitz, T. Lorenz, and M. M. Abd-Elmeguid, Phys. Rev. B 69, 140403 (2004).

[14] I. Fita, R. Szymczak, R. Puzniak, I. O. Troyanchuk, J. Fink-Finowicki, Ya. M. Mukovskii, V. N. Varyukhin, and H. Szymczak, Phys. Rev. B 71, 214404 (2005).

[15] M. A. Senaris-Rodriguez, and J. B. Goodenough, J. Solid State Chem. 116, 224 (1995).

[16] S. Tsubouchi, T. Kyômen, M. Itoh, P. Ganguly, M. Oguni, Y. Shimojo, Y. Morii, and Y. Yoshii, Phys. Rev. B 66, 052418 (2002). 
[17] S. Tsubouchi, T. Kyômen, and M. Itoh, Phys. Rev. B 69, 144406 (2004).

[18] M. Kriener, C. Zobel, A. Reichl, J. Baier, M. Cwik, K. Berggold, H. Kierspel, O. Zabara, A. Freimuth, and T. Lorenz, Phys. Rev. B 69, 094417 (2004).

[19] X. G. Luo et al., cond-mat/0501562 (unpublished).

[20] A. Maignan, C. Martin, N. Nguyen, and B. Raveau, Solid State Sciences, 3, 57 (2001).

[21] R. P Haggerty and R. Seshadri, J. Phys.: Condens. Matt. 16, 6477 (2004).

[22] T. Fujita, S. Kawabata, M. Sato, K. Kakurai, M. Hedo, and Y. Uwatoko, cond-mat/0502631 (unpublished).

[23] R. D. Shannon, Acta Crystallogr. A32, 751 (1976).

[24] R. D. Shannon, C. T. Prewitt, Acta Crystallogr. B25, 925 (1969).

[25] Y. L. Chang, Q. Huang, and C. K. Ong, J. Appl. Phys. 91, 789 (2002).

[26] T. Fujita, T. Miyashita, Y. Yasui, Y. Kobayashi, M. Sato, E. Nishibori, M. Sakata, Y. Shimojo, N. Igawa, Y. Ishii, K. Kakurai, T. Adachi, Y. Ohishi, M. Takata, J. Phys. Soc. Jpn, 73, 1987 (2004).

[27] M. Katsuki, S. R. Wang, M. Dokiya, T. Hashimoto, Solid State Ionics 156, 453 (2003). S. R. Wang, M. Katsuki, M. Dokiya, T. Hashimoto, ibid 159, 71 (2003).

[28] M. Kopcewicz, D. D. Khalyavin, I. O. Troyanchuk, H. Szymczak, R. Szymczak, D. J. Logvinovich, and E. N. Naumovich, J. Appl. Phys. 93, 479 (2003).

[29] Á. Cziráki, I. Geröcs, M. Köteles, A. Gábris, L. Pogány, I. Bakonyi, Z. Klencsár, A. Vértes, S. K. De, A. Barman, M. Ghosh, S. Biswas, S. Chatterjee, B. Arnold, H. D. Bauer, K. Wetzig, C. Ulhaq-Bouillet, and V. Pierron- Bohnes, Eur. Phys. J. B 21, 521 (2001).

[30] Z. Homonnay, E. Kuzmann, Z. Nemeth, Z. Klencsar, S. I. Nagy, and A. Vertes, CeramicsSilikaty 48, 197 (2004). 


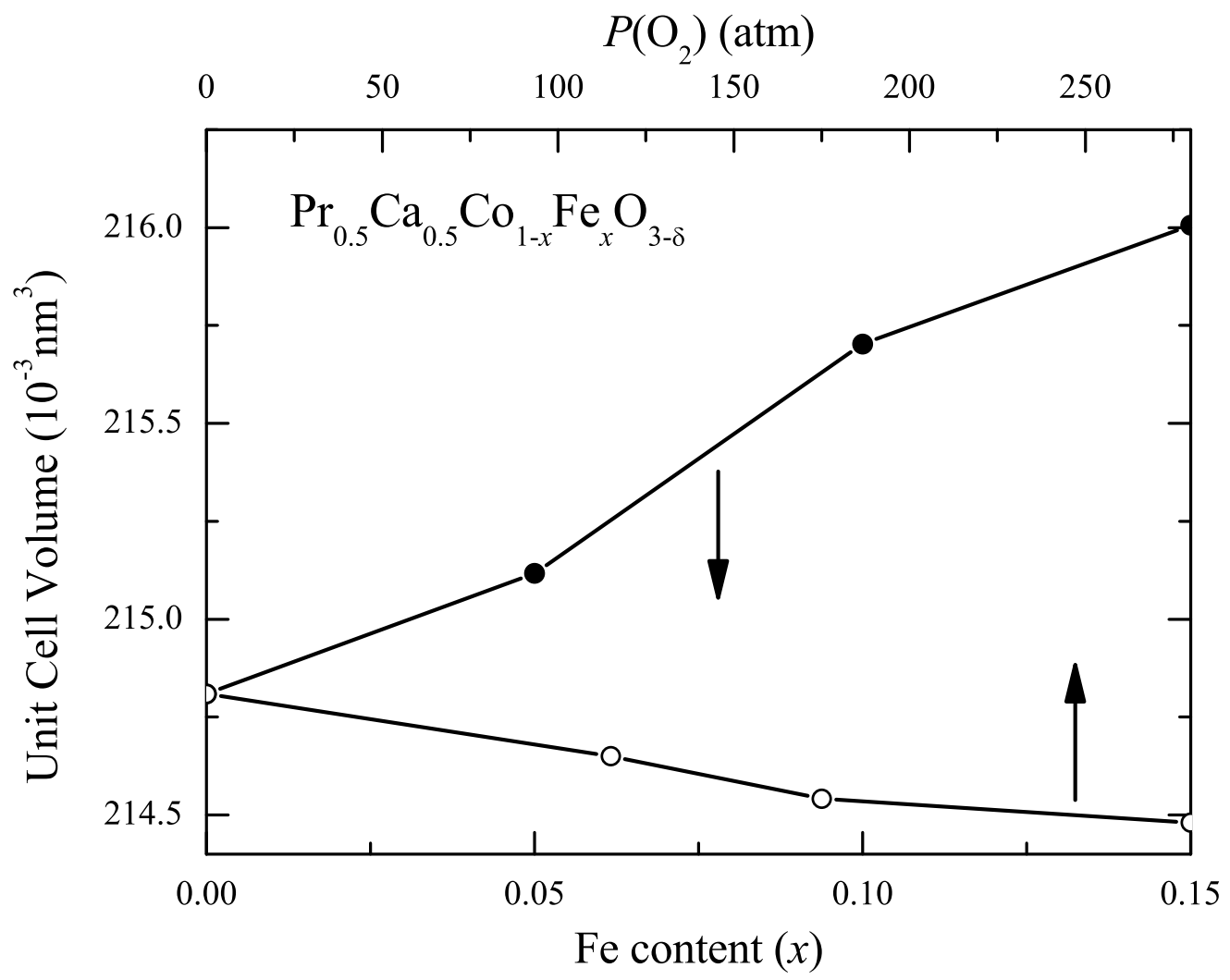

FIG. 1: The unit cell volume variation with $x$ for the as-fabricated $\operatorname{Pr}_{0.5} \mathrm{Ca}_{0.5} \mathrm{Co}_{1-x} \mathrm{Fe}_{x} \mathrm{O}_{3-\delta}$ polycrystals. 


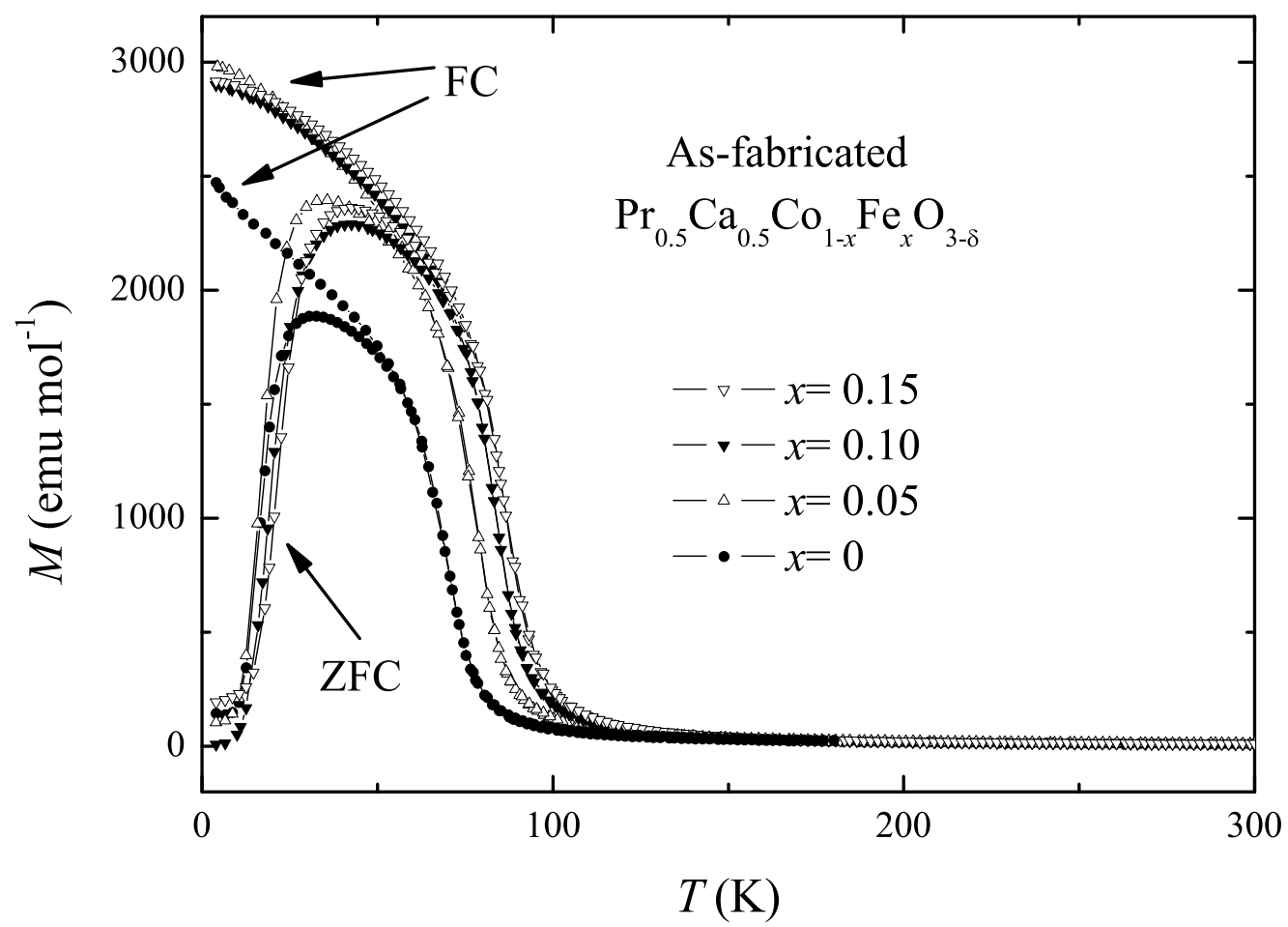

FIG. 2: The temperature dependence of the molar magnetization recorded at $H=0.1 \mathrm{~T}$ for the as-fabricated $\operatorname{Pr}_{0.5} \mathrm{Ca}_{0.5} \mathrm{Co}_{1-x} \mathrm{Fe}_{x} \mathrm{O}_{3-\delta}$ polycrystals. 


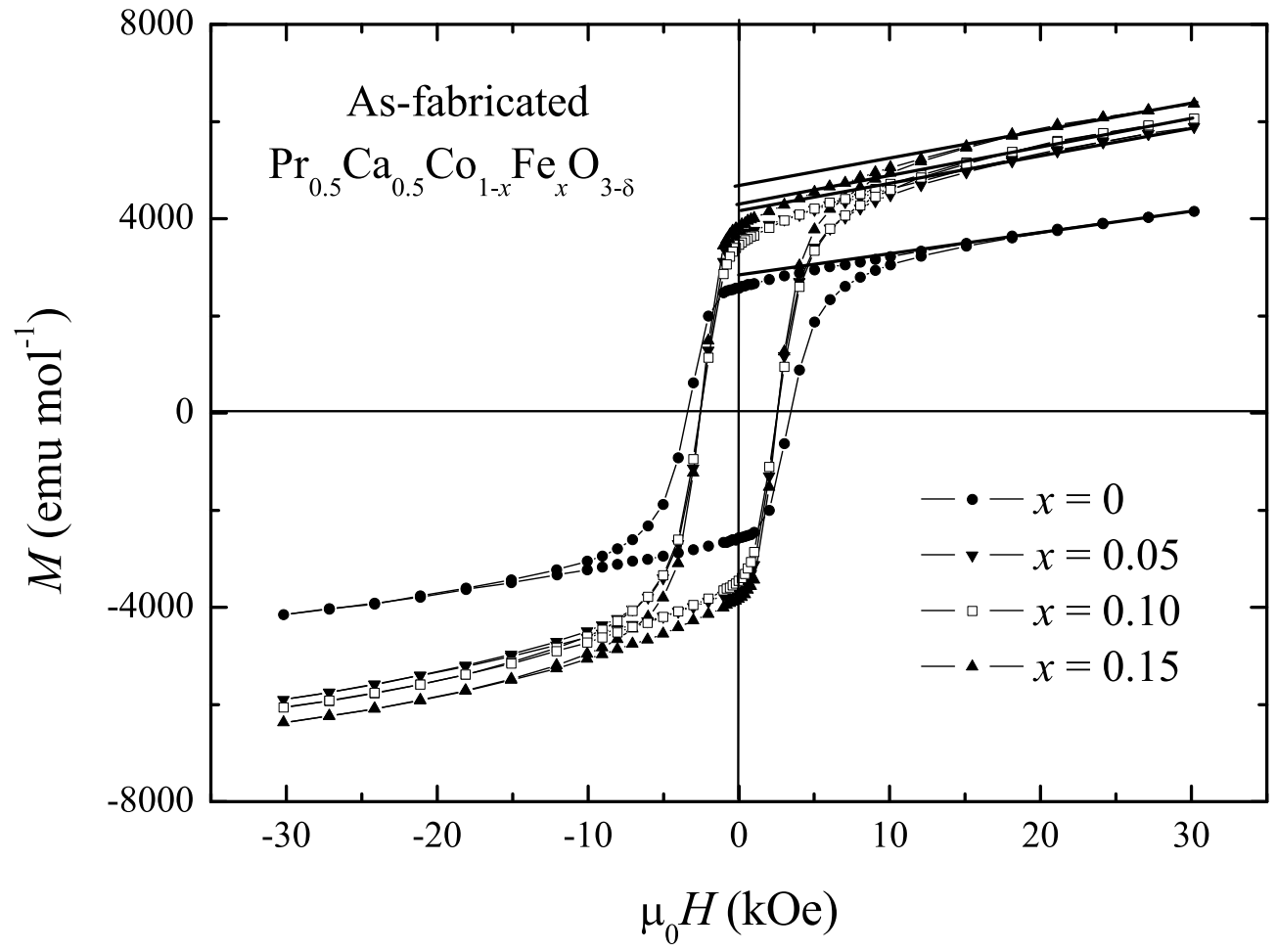

FIG. 3: The $M-H$ loop at $4 \mathrm{~K}$ for the as-fabricated $\mathrm{Pr}_{0.5} \mathrm{Ca}_{0.5} \mathrm{Co}_{1-x} \mathrm{Fe}_{x} \mathrm{O}_{3-\delta}$ polycrystals. The solid lines extrapolate the $M(H)$ to $H=0$ to determined the spontaneous magnetization. 


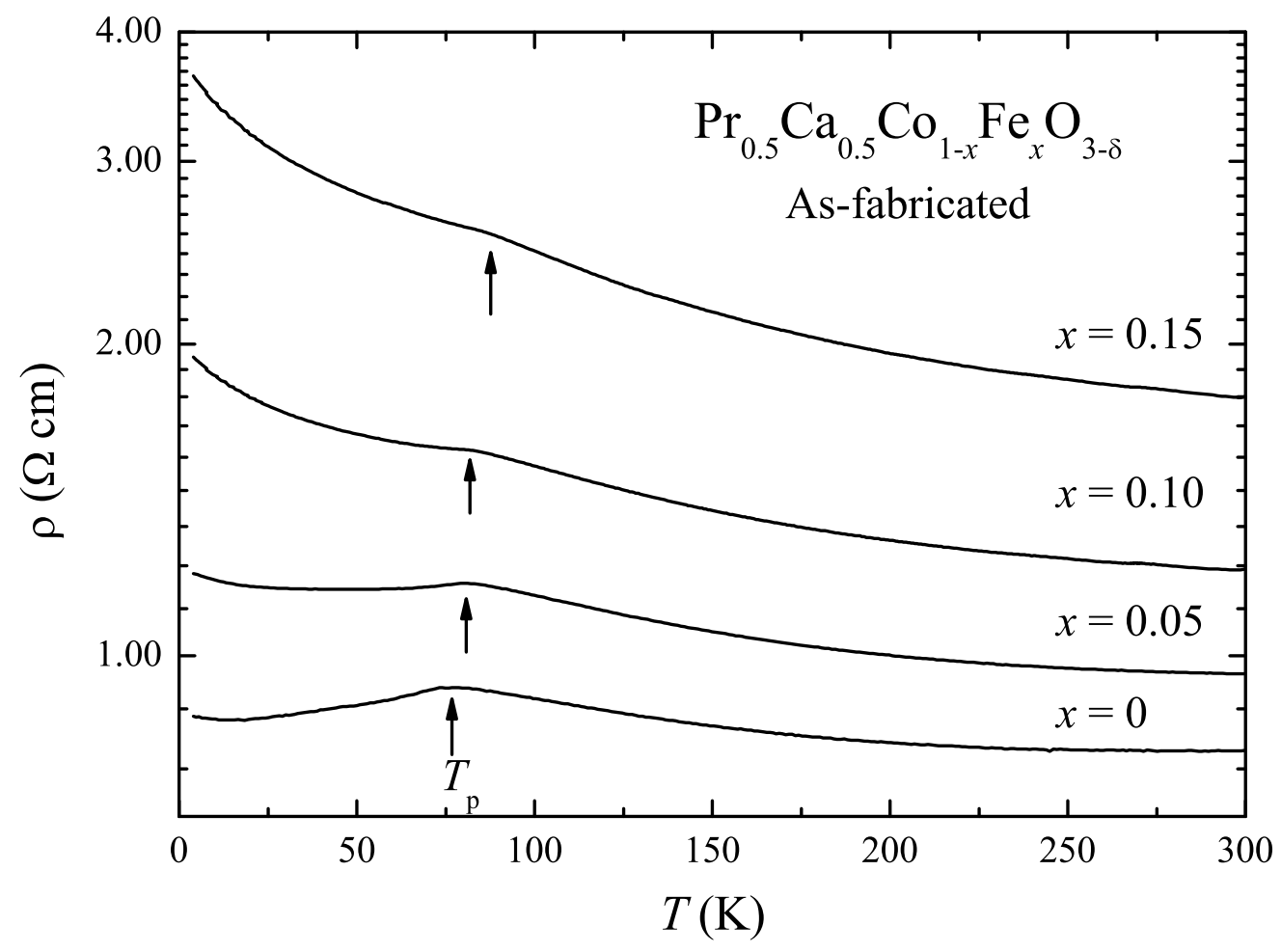

FIG. 4: The temperature dependence of the resistivity for the as-fabricated $\operatorname{Pr}_{0.5} \mathrm{Ca}_{0.5} \mathrm{Co}_{1-x} \mathrm{Fe}_{x} \mathrm{O}_{3-\delta}$ polycrystals. The dashed arrows point to the $T_{\mathrm{p}}$. 


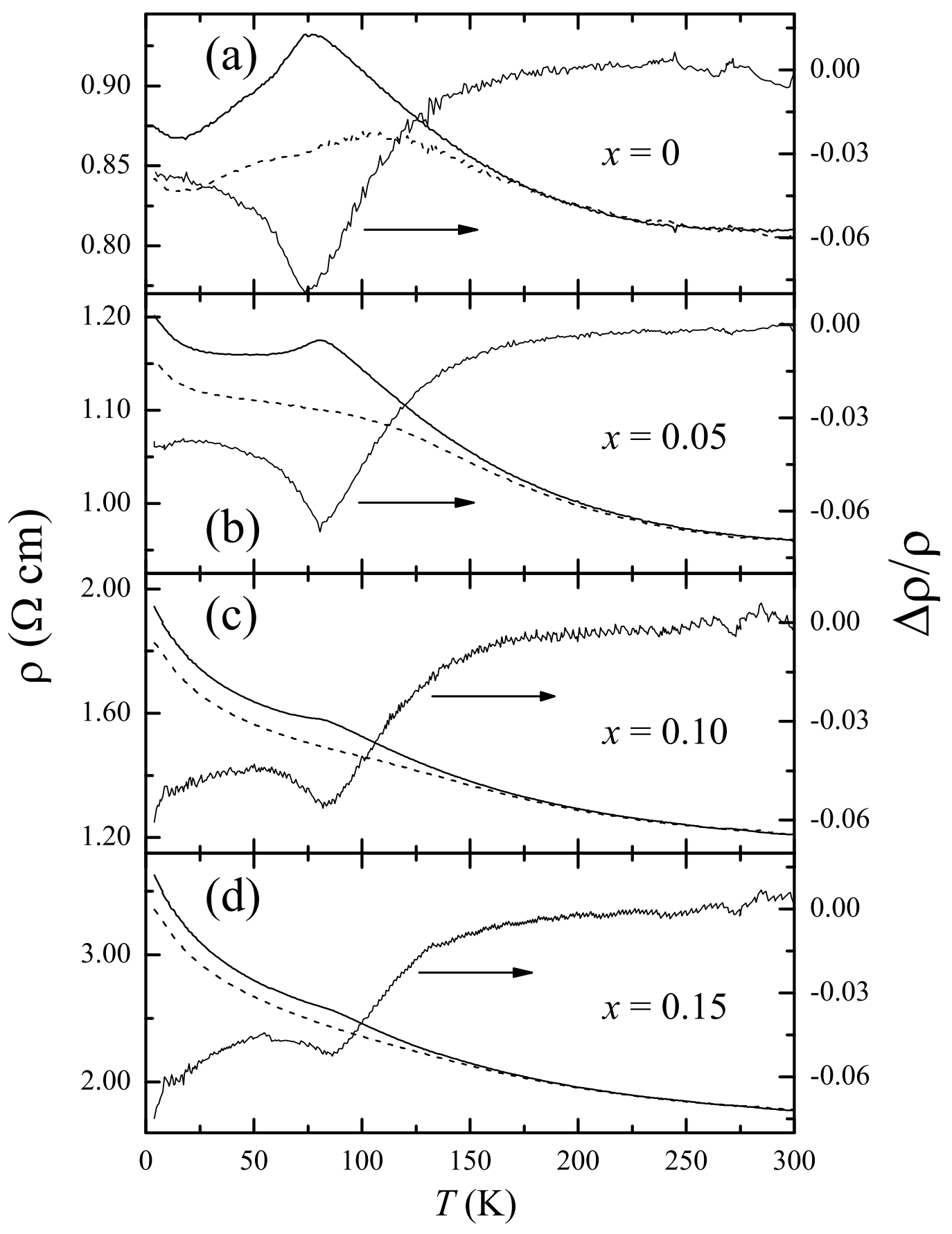

FIG. 5: Resistivity collected at $H=0$ (solid lines) and $6 \mathrm{~T}$ (dash lines) and magnetoresistance $(\Delta \rho / \rho)$ at $H=6 \mathrm{~T}$ as the function of the temperature for the as-fabricated $\operatorname{Pr}_{0.5} \mathrm{Ca}_{0.5} \mathrm{Co}_{1-x} \mathrm{Fe}_{x} \mathrm{O}_{3-\delta}$ polycrystals. 


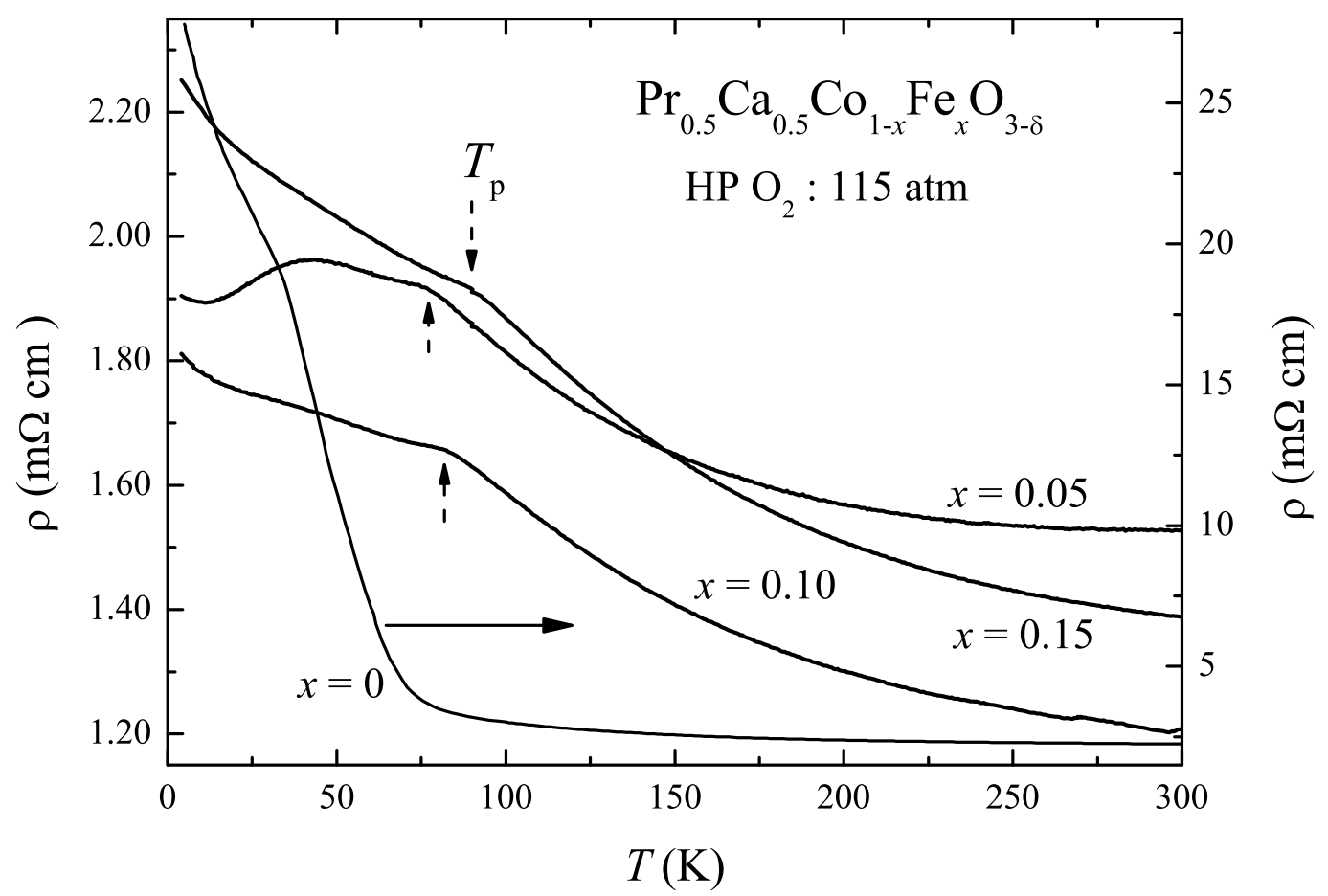

FIG. 6: The temperature dependence of the resistivity for the $\mathrm{Pr}_{0.5} \mathrm{Ca}_{0.5} \mathrm{Co}_{1-x} \mathrm{Fe}_{x} \mathrm{O}_{3-\delta}$ polycrystals after annealing at $600^{\circ} \mathrm{C}$ and under the oxygen pressure of $115 \mathrm{~atm}$. The dashed arrows point to the $T_{\mathrm{p}}$. 


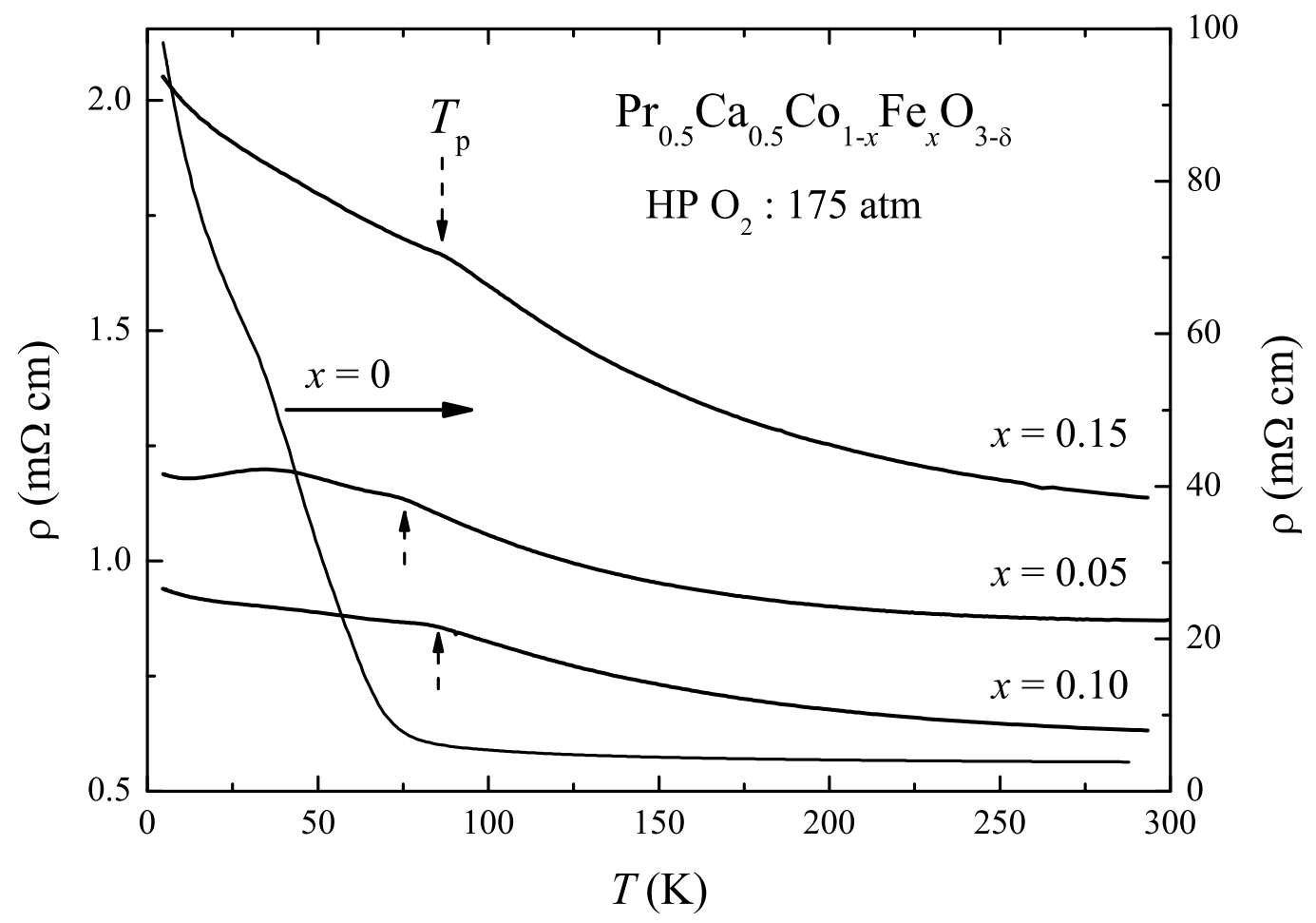

FIG. 7: The temperature dependence of the resistivity for the $\operatorname{Pr}_{0.5} \mathrm{Ca}_{0.5} \mathrm{Co}_{1-x} \mathrm{Fe}_{x} \mathrm{O}_{3-\delta}$ polycrystals after annealing at $600^{\circ} \mathrm{C}$ for $48 \mathrm{~h}$ under the oxygen pressure of $175 \mathrm{~atm}$. The dashed arrows point to the $T_{\mathrm{p}}$. 


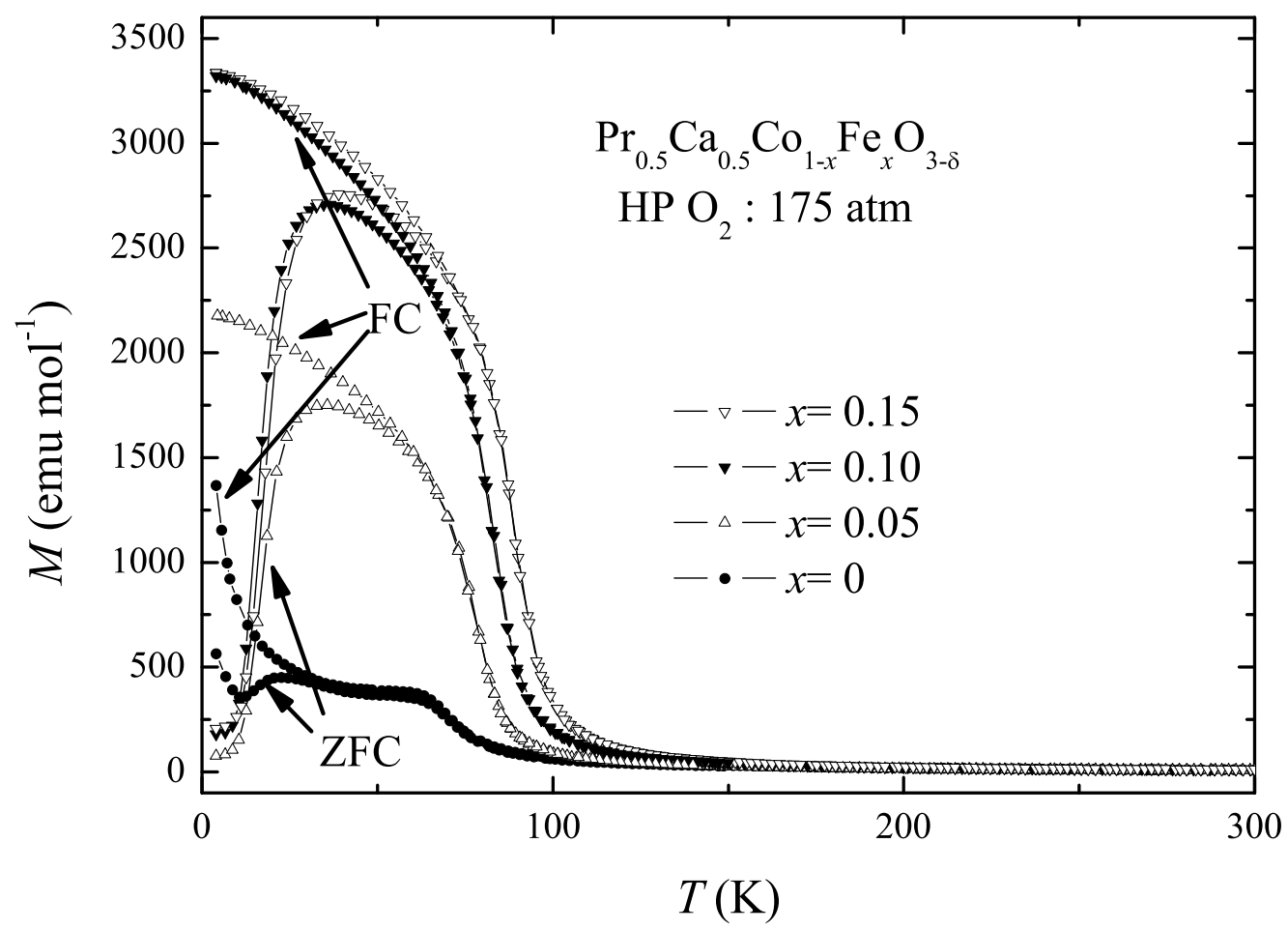

FIG. 8: The temperature dependence of the molar magnetization recorded at $H=0.1 \mathrm{~T}$ for the $\operatorname{Pr}_{0.5} \mathrm{Ca}_{0.5} \mathrm{Co}_{1-x} \mathrm{Fe}_{x} \mathrm{O}_{3-\delta}$ polycrystals after annealing at $600^{\circ} \mathrm{C}$ for $48 \mathrm{~h}$ under the oxygen pressure of 175 atm. 


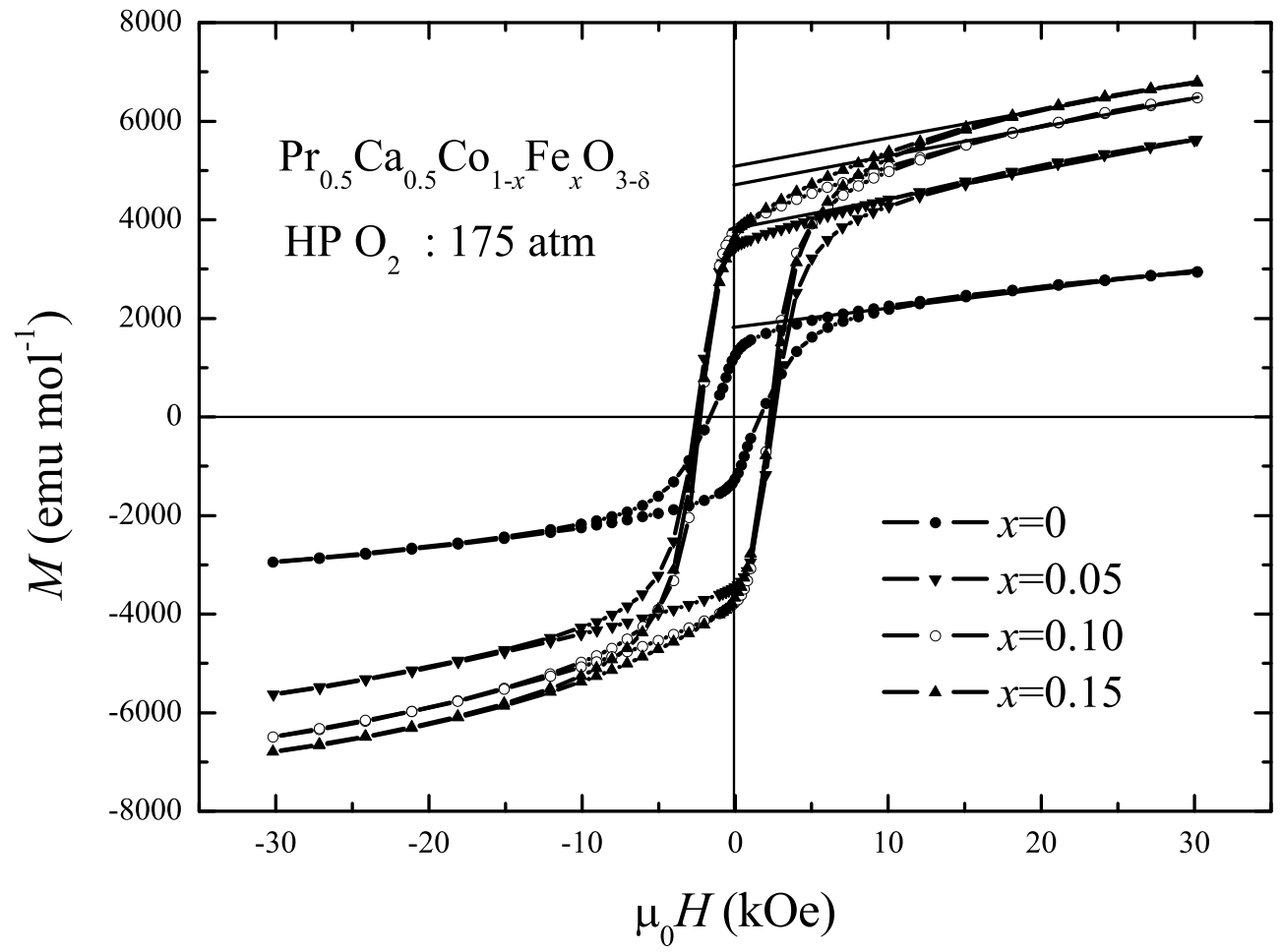

FIG. 9: $M-H$ loops at $4 \mathrm{~K}$ for the $\operatorname{Pr}_{0.5} \mathrm{Ca}_{0.5} \mathrm{Co}_{1-x} \mathrm{Fe}_{x} \mathrm{O}_{3-\delta}$ polycrystals after annealing at $600{ }^{\circ} \mathrm{C}$ for $48 \mathrm{~h}$ under the oxygen pressure of $175 \mathrm{~atm}$. The solid lines extrapolate the $M(H)$ to $H=0$ to determined the spontaneous magnetization. 


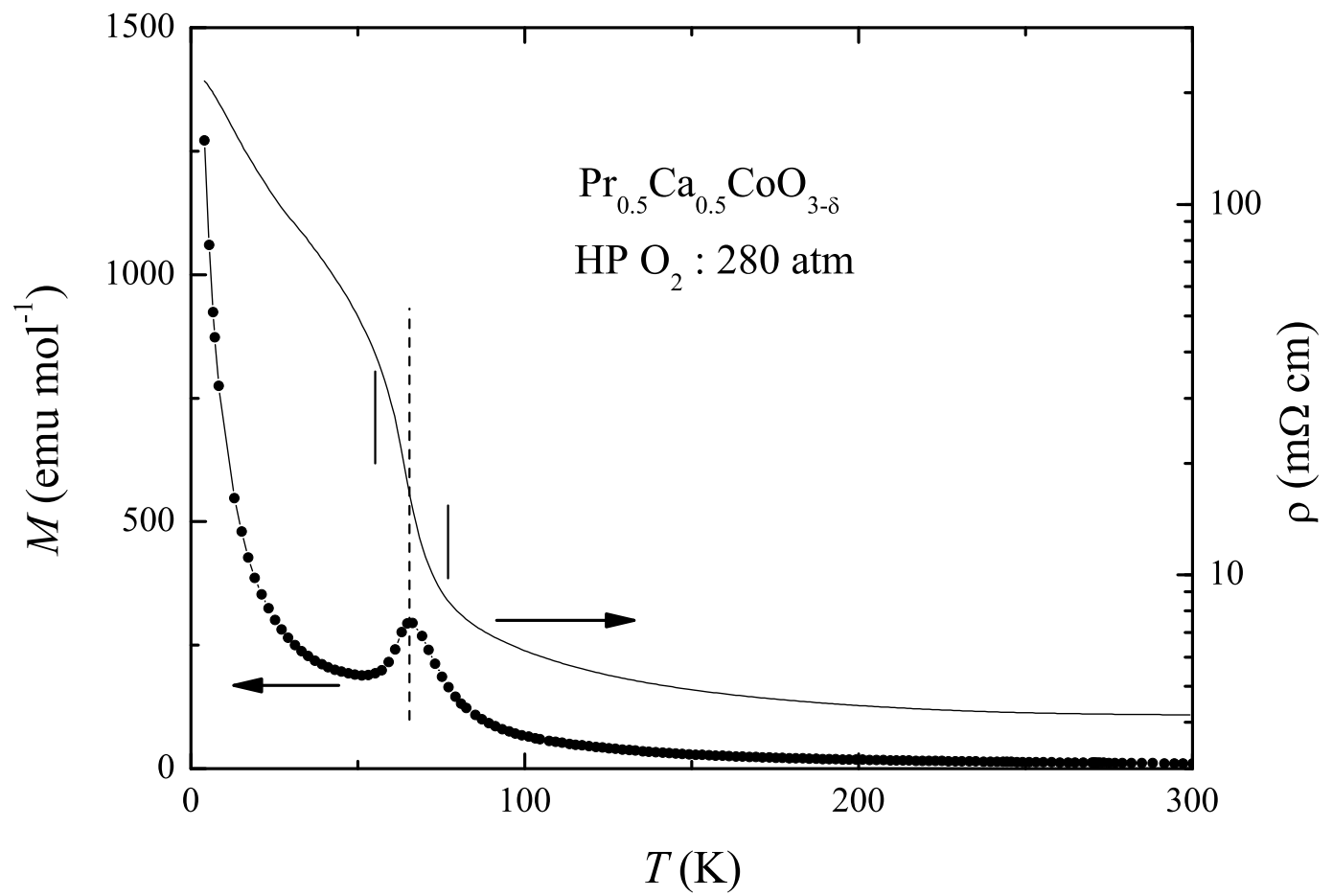

FIG. 10: The FC molar magnetization recorded at $H=0.1 \mathrm{~T}$ and the resistivity at zero field as the function temperature for the $\operatorname{Pr}_{0.5} \mathrm{Ca}_{0.5} \mathrm{CoO}_{3-\delta}$ polycrystals after annealing at $600^{\circ} \mathrm{C}$ and under the oxygen pressure of $280 \mathrm{~atm}$. 


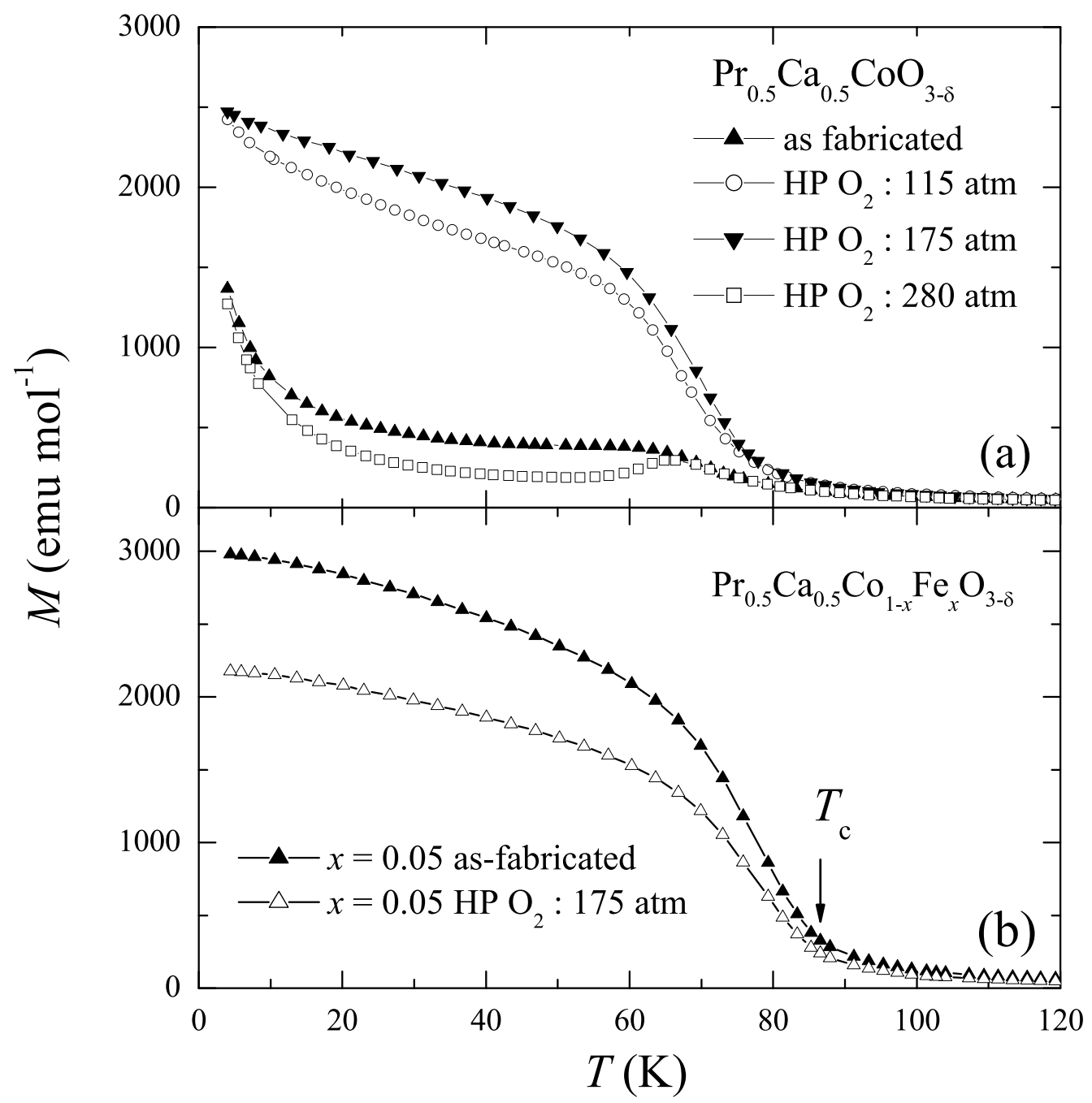

FIG. 11: (a)The FC molar magnetization at $H=0.1 \mathrm{~T}$ as the function temperature for the $\operatorname{Pr}_{0.5} \mathrm{Ca}_{0.5} \mathrm{CoO}_{3-\delta}$ polycrystals obtained through different conditions.(b)The temperature dependence of the FC molar magnetization at $H=0.1 \mathrm{~T}$ for the as-fabricated and the annealed $\operatorname{Pr}_{0.5} \mathrm{Ca}_{0.5} \mathrm{Co}_{0.95} \mathrm{Fe}_{0.05} \mathrm{O}_{3-\delta}$. 